\title{
The Effect of the COVID-19 Pandemic on Health Care Workers' Anxiety Levels: Protocol for a Meta-Analysis
}

Lunbo Zhang ${ }^{1}$, BS; Ming Yan ${ }^{1}$, BS; Kaito Takashima ${ }^{1}$, BA; Wenru Guo ${ }^{1}$, BS; Yuki Yamada ${ }^{2}$, PhD

${ }^{1}$ Graduate School of Human-Environment Studies, Kyushu University, Fukuoka, Japan

${ }^{2}$ Faculty of Arts and Science, Kyushu University, Fukuoka, Japan

Corresponding Author:

Lunbo Zhang, BS

Graduate School of Human-Environment Studies

Kyushu University

744 Motooka, Nishi-ku

Fukuoka, 819-0395

Japan

Phone: 810928025251

Email: zhanglunbo014@gmail.com

\section{Abstract}

Background: The COVID-19 pandemic has been declared a public health emergency of international concern; this has caused excessive anxiety among health care workers. In addition, publication bias and low-quality publications have become widespread, which can result in the dissemination of unreliable findings.

Objective: This paper presents the protocol for a meta-analysis with the following two aims: (1) to examine the prevalence of anxiety among health care workers and determine whether it has increased due to the COVID-19 pandemic, and (2) to investigate whether there has been an increase in publication bias.

Methods: All related studies that were published/released from 2015 to 2020 will be searched in electronic databases (Web of Science, PubMed, PsyArXiv, and medRxiv). The risk of bias in individual studies will be assessed using the STROBE (Strengthening the Reporting of Observational Studies in Epidemiology) checklist. The heterogeneity of the studies will be assessed using the $\mathrm{I}^{2}$ statistic. The effect size (prevalence rates of anxiety) and a 95\% CI for each paper will also be calculated. We will use a moderator analysis to test for the effect of COVID-19 on health care workers' anxiety levels and detect publication bias in COVID-19 studies. We will also assess publication bias using the funnel plot and Egger regression. In case of publication bias, if studies have no homogeneity, the trim-and-fill procedure will be applied to adjust for missing studies.

Results: Database searches will commence in November 2020. The meta-analysis will be completed within 2 months of the start date.

Conclusions: This meta-analysis aims to provide comprehensive evidence about whether COVID-19 increases the prevalence of anxiety among health care workers and whether there has been an increase in publication bias and a deterioration in the quality of publications due to the pandemic. The results of this meta-analysis can provide evidence to help health managers to make informed decisions related to anxiety prevention in health care workers.

International Registered Report Identifier (IRRID): PRR1-10.2196/24136

(JMIR Res Protoc 2020;9(11):e24136) doi: $\underline{10.2196 / 24136}$

\section{KEYWORDS}

COVID-19; health care worker; anxiety; meta-analysis; review; protocol; mental health; literature; bias

\section{Introduction}

\section{Background and Research Questions}

The COVID-19 pandemic has affected more than 18.9 million individuals and resulted in over 709,000 deaths globally [1]. It has, therefore, been declared a public health emergency of international concern [2]. To tide over this crisis, it is important to maintain an adequate health care workforce, which requires not only an sufficient number of health care workers but also the maximization of each health care worker's ability to care for a greater number of patients. Since the outbreak can last 
several months, it is also critical that health care workers are able to perform to their full potential over an extended time interval [3].

The COVID-19 pandemic has affected many aspects of people's lives, especially their mental health [4-7]. While health care workers have to concurrently cope with the societal shifts and emotional stressors faced by the general population, they additionally face greater risks of exposure, extreme workloads, moral dilemmas, and rapidly evolving practice environments that differ greatly from what they are familiar with $[8,9]$. Moreover, facing hitherto unknown challenges in both physical and mental health causes excessive tension and anxiety in health care workers [10]. While anxiety is a common mental condition that can cause emotional distress, obsessive thinking, and compulsive behavior, long-term anxiety results in psychological distress and even affects the daily lives of individuals [11]. Anxiety also impairs the executive functions that underlie our ability to control and focus on our thoughts [12]. Consequently, studying and accurately grasping the anxiety levels of health care workers is necessary to take more appropriate and corrective measures to deal with public health and safety.

Although some researchers have investigated health care workers' anxiety levels during the COVID-19 pandemic [13,14], many new papers on COVID-19 are being released rapidly since the pandemic still poses a serious threat. The present meta-analytic study includes the latest papers, and aims to generate a more comprehensive understanding of the prevalence of anxiety among health care workers. Furthermore, to date, a comparison has not been established between studies on health care workers' anxiety levels, related and unrelated to COVID-19. In the current outbreak situation, will studies conducted in two different periods have different effect sizes? Will levels of anxiety increase significantly? Accordingly, the first aim of our meta-analysis is to examine health care workers' anxiety status and determine the COVID-19 pandemic's influence by comparing COVID-19-related studies with unrelated studies.

In addition, since the onset of the outbreak, knowledge about COVID-19 is direly needed, and medical journals have drastically accelerated the publication process for COVID-19-related articles to accelerate knowledge acquisition $[15,16]$. In this situation, the preference for publishing papers with significant results may be more extreme, which may seriously compromise the ability to draw valid conclusions from the published literature. Since the publication bias may be highly flawed, the second aim of our meta-analysis is to investigate publication bias by comparing unpublished preprints on COVID-19 with published journal papers about COVID-19.

\section{Hypotheses}

We have generated the following two hypotheses:

1. COVID-19 makes health care workers more anxious and thus the studies related to COVID-19 will have a larger effect size. We will investigate this by comparing studies related to COVID-19 vs studies unrelated to it.

2. Publication bias in COVID-19-related studies is widespread. We will investigate this by comparing unpublished preprints about COVID-19 with published journal papers about the disease.

\section{Methods}

\section{Search Strategy}

This study will follow the PRISMA (Preferred Reporting Items for Systematic Reviews and Meta-Analyses) guidelines [17]. We will search through electronic databases-Web of Science, PubMed, PsyArXiv, and medRxiv—for all published journal papers (related vs unrelated to COVID-19) and preprints (relevant to COVID-19), whose titles and abstracts include the search terms presented in Textbox 1.

Textbox 1. Search terms.

("Health Personnel" OR "Personnel, Health" OR "Health Care Providers" OR "Health Care Provider" OR "Provider, Health Care" OR "Providers, Health Care" OR "Healthcare Providers" OR "Healthcare Provider" OR "Provider, Healthcare" OR "Providers, Healthcare" OR "Healthcare Workers" OR "Healthcare Worker" OR "Health Occupations" OR "Health Occupation" OR "Health Professions" OR "Health Profession" OR "Profession, Health" OR "Professions, Health" OR "Health professions")

AND

(Anxiety OR Hypervigilance OR Nervousness OR “Social Anxiety” OR “Anxieties, Social” OR "Anxiety, Social” OR "Social Anxieties”)

\section{Inclusion and Exclusion Criteria}

Studies will be included only if they meet the following inclusion criteria: (1) written in English (which will be decided based on the research team's unified considerations); (2) related to "anxiety among health care workers"; (3) quantitative research designs; (4) submitted during 2015 to 2020; (5) include standardized measures of anxiety with published psychometric data and reasonable evidence of reliability and validity; (6) include a clear description of methods used to assess and score standardized measurement instruments; and (7) include publicly available effect sizes (prevalence) or values that can be calculated (the number of health care workers with anxiety and the sample size).
The exclusion criteria are: (1) studies with insufficient data, (2) duplicate sources, (3) research with unclear methods, and (4) publications about other outbreaks.

\section{Data Extraction}

First, duplicate papers that are found in multiple databases will be removed. Subsequently, screening of the titles and abstracts will be conducted, and papers will be removed based on the inclusion and exclusion criteria. Furthermore, the full text of the papers will be checked, and article information will be extracted using a preprepared extraction table that includes the article's title, authors' names, scales used, year of submission, country, sample size, whether the study has been published, whether the study relates to COVID-19, and the effect size 
(prevalence of anxiety). The article review and data extraction processes will be performed independently by two of the authors. When there is a disagreement between them, the other authors will resolve the conflict.

\section{Study Assessment Criteria}

We will use the STROBE (Strengthening the Reporting of Observational Studies in Epidemiology) checklist to assess the quality of observational studies [18]. The checklist consists of 6 scales-title, abstract, introduction, method, results, and discussion-each of which includes multiple items, comprising a total of 32 items. Each item is scored as 0 (not fulfilled) or 1 (fulfilled). In the modified STROBE, scores range from 0 to 32 , with scores $\geq 16$ indicating a low risk of bias and scores $<16$ indicating a high risk of bias. Papers that exhibit a low risk of bias will be selected for the analysis.

\section{Statistical Analysis}

First, the heterogeneity of the studies will be determined using the $\mathrm{I}^{2}$ statistical index, which ranges from 0 to 100; the larger the index, the more heterogeneous are the findings. The categories encompassed by the index will be defined based on the test developed by Higgins et al [19] to measure the extent of heterogeneity: low (25\%), moderate (50\%), and high $(75 \%)$. A study with a heterogeneity $>50 \%$ prompts the use of random effects models. For each research, we will calculate the effect size (prevalence rates of anxiety) and a 95\% CI around the effect size. For the data reported, if the original paper does not list the effect size or the number of health care workers with anxiety (which can be used to calculate the effect size), the authors of the paper will be contacted and asked to provide this information. If they are unable to do so, the study will be excluded from the analyses.

Subsequently, we will use a moderator analysis to test for the effect of COVID-19 on health care workers' anxiety levels (related vs unrelated to COVID-19), and publication bias in COVID-19 studies (preprints vs published journal papers). We will also assess publication bias using the funnel plot and Egger regression [20]. For the Egger regression, a $P$ value less than the significance level $(\alpha=.05)$ suggests that publication bias is present. If publication bias is present, and studies have no homogeneity, the trim-and-fill procedure will be applied to adjust these missing studies [21].

Finally, sensitivity analyses will be performed to assess the influence of each individual study on the pooled effect size. The statistical significance level is defined as $\alpha=.05$.

\section{Results}

Database searches will commence in November 2020. The meta-analysis will be completed within 2 months.

\section{Discussion}

This paper presents a protocol for a meta-analysis that aims to provide comprehensive evidence about whether the COVID-19 pandemic increases the prevalence of anxiety among health care workers and whether there has been an increase in publication bias and a deterioration in the quality of publications due to the pandemic. The results of this meta-analysis can provide evidence to help health managers to make informed decisions for preventing anxiety in health care workers.

\section{Acknowledgments}

This research was supported by the Japan Society for the Promotion of Science KAKENHI (16H03079, 17H00875, 18K12015, and 20H04581).

\section{Conflicts of Interest}

None declared.

\section{References}

1. Coronavirus disease (COVID-19) Situation Report - 200. World Health Organization. 2020. URL: https://www.who.int/ docs/default-source/coronaviruse/situation-reports/20200807-covid-19-sitrep-200.pdf?sfvrsn=2799bc0f 2 [accessed 2020-08-08]

2. COVID-19 Public Health Emergency of International Concern (PHEIC) Global research and innovation forum. World Health Organization. 2020. URL: https://www.who.int/publications/m/item/ covid-19-public-health-emergency-of-international-concern-(pheic)-global-research-and-innovation-forum [accessed 2020-08-17]

3. Shanafelt T, Ripp J, Trockel M. Understanding and Addressing Sources of Anxiety Among Health Care Professionals During the COVID-19 Pandemic. JAMA 2020 Jun 02;323(21):2133-2134. [doi: 10.1001/jama.2020.5893] [Medline: 32259193]

4. Wang C, Pan R, Wan X, Tan Y, Xu L, Ho CS, et al. Immediate psychological responses and associated factors during the initial stage of the 2019 coronavirus disease (COVID-19) epidemic among the general population in China. Int J Environ Res Public Health 2020 Mar 06;17(5):1439-1440 [FREE Full text] [doi: 10.3390/ijerph17051729] [Medline: $\underline{\text { 32155789] }}$

5. Özdin S, Bayrak Özdin Ş. Levels and predictors of anxiety, depression and health anxiety during COVID-19 pandemic in Turkish society: The importance of gender. Int J Soc Psychiatry 2020 Aug;66(5):504-511 [FREE Full text] [doi: 10.1177/0020764020927051] [Medline: 32380879] 
6. Mazza C, Ricci E, Biondi S, Colasanti M, Ferracuti S, Napoli C, et al. A Nationwide Survey of Psychological Distress among Italian People during the COVID-19 Pandemic: Immediate Psychological Responses and Associated Factors. Int J Environ Res Public Health 2020 May 02;17(9) [FREE Full text] [doi: 10.3390/ijerph17093165] [Medline: $\underline{\text { 32370116] }}$

7. González-Sanguino C, Ausín B, Castellanos M, Saiz J, López-Gómez A, Ugidos C, et al. Mental health consequences during the initial stage of the 2020 Coronavirus pandemic (COVID-19) in Spain. Brain Behav Immun 2020 Jul;87:172-176 [FREE Full text] [doi: 10.1016/j.bbi.2020.05.040] [Medline: 32405150]

8. Adams JG, Walls RM. Supporting the Health Care Workforce During the COVID-19 Global Epidemic. JAMA 2020 Apr 21;323(15):1439-1440. [doi: 10.1001/jama.2020.3972] [Medline: 32163102]

9. Xiang Y, Yang Y, Li W, Zhang L, Zhang Q, Cheung T, et al. Timely mental health care for the 2019 novel coronavirus outbreak is urgently needed. Lancet Psychiatry 2020 Mar;7(3):228-229 [FREE Full text] [doi:

10.1016/S2215-0366(20)30046-8] [Medline: 32032543]

10. Albott CS, Wozniak JR, McGlinch BP, Wall MH, Gold BS, Vinogradov S. Battle Buddies: Rapid Deployment of a Psychological Resilience Intervention for Health Care Workers During the COVID-19 Pandemic. Anesth Analg 2020 Jul;131(1):43-54 [FREE Full text] [doi: 10.1213/ANE.0000000000004912] [Medline: 32345861]

11. Cole AH. Anxiety. In: Leeming DA, editor. Encyclopedia of Psychology and Religion. Boston, MA: Springer; 2014:95-99.

12. Shields GS, Moons WG, Tewell CA, Yonelinas AP. The effect of negative affect on cognition: Anxiety, not anger, impairs executive function. Emotion 2016 Sep;16(6):792-797 [FREE Full text] [doi: 10.1037/emo0000151] [Medline: 27100367]

13. Pappa S, Ntella V, Giannakas T, Giannakoulis VG, Papoutsi E, Katsaounou P. Prevalence of depression, anxiety, and insomnia among healthcare workers during the COVID-19 pandemic: A systematic review and meta-analysis. Brain Behav Immun 2020 May 08 [FREE Full text] [doi: 10.1016/j.bbi.2020.05.026] [Medline: $\underline{\text { 32437915] }}$

14. Pan R, Zhang L, Pan J. The Anxiety Status of Chinese Medical Workers During the Epidemic of COVID-19: A Meta-Analysis. Psychiatry Investig 2020 May;17(5):475-480 [FREE Full text] [doi: 10.30773/pi.2020.0127] [Medline: 32403209]

15. Palayew A, Norgaard O, Safreed-Harmon K, Andersen TH, Rasmussen LN, Lazarus JV. Pandemic publishing poses a new COVID-19 challenge. Nat Hum Behav 2020 Jul 23;4(7):666-669. [doi: 10.1038/s41562-020-0911-0] [Medline: 32576981]

16. Horbach SPJM. Pandemic publishing: Medical journals strongly speed up their publication process for COVID-19. bioRxiv Preprint posted online April 18, 2020. [doi: 10.1101/2020.04.18.045963]

17. Moher D, Liberati A, Tetzlaff J, Altman DG. Preferred reporting items for systematic reviews and meta-analyses: the PRISMA statement. PLoS Med 2009 Jul 21;6(7):e1000097 [FREE Full text] [doi: 10.1371/journal.pmed.1000097] [Medline: 19621072]

18. Sanderson S, Tatt ID, Higgins JPT. Tools for assessing quality and susceptibility to bias in observational studies in epidemiology: a systematic review and annotated bibliography. Int J Epidemiol 2007 Jun;36(3):666-676 [FREE Full text] [doi: $10.1093 / \mathrm{ije} / \mathrm{dym} 018]$ [Medline: 17470488$]$

19. Higgins JPT, Thompson SG, Deeks JJ, Altman DG. Measuring inconsistency in meta-analyses. BMJ 2003 Sep 6;327(7414):557-560 [FREE Full text] [doi: 10.1136/bmj.327.7414.557] [Medline: 12958120]

20. Egger M, Davey Smith G, Schneider M, Minder C. Bias in meta-analysis detected by a simple, graphical test. BMJ 1997 Sep 13;315(7109):629-634 [FREE Full text] [doi: 10.1136/bmj.315.7109.629] [Medline: 9310563]

21. Duval S, Tweedie R. A Nonparametric “Trim and Fill” Method of Accounting for Publication Bias in Meta-Analysis. Journal of the American Statistical Association 2000 Mar;95(449):89-98. [doi: 10.1080/01621459.2000.10473905]

\section{Abbreviations}

PRISMA: Preferred Reporting Items for Systematic Reviews and Meta-Analyses

STROBE: Strengthening the Reporting of Observational Studies in Epidemiology

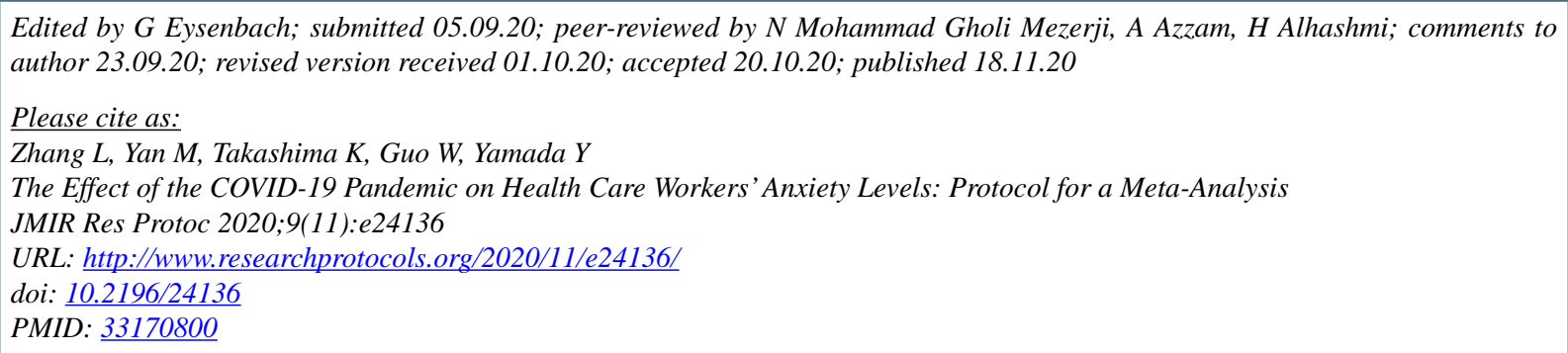


CLunbo Zhang, Ming Yan, Kaito Takashima, Wenru Guo, Yuki Yamada. Originally published in JMIR Research Protocols (http://www.researchprotocols.org), 18.11.2020. This is an open-access article distributed under the terms of the Creative Commons Attribution License (https://creativecommons.org/licenses/by/4.0/), which permits unrestricted use, distribution, and reproduction in any medium, provided the original work, first published in JMIR Research Protocols, is properly cited. The complete bibliographic information, a link to the original publication on http://www.researchprotocols.org, as well as this copyright and license information must be included. 